\title{
Centro de Especialidade Odontológica e Unidade Básica de Saúde: análise de Redes Sociais como estratégia de governança
}

\author{
Fabio Rocha de Paula ${ }^{a^{*}}$, Marcia Gomide da Silva Mello ${ }^{\mathrm{b}}$ \\ ${ }^{\text {a} P r o g r a m a ~ d e ~ P o ́ s ~ G r a d u a c ̧ a ̃ o ~ e m ~ S a u ́ d e ~ C o l e t i v a, ~ U n i v e r s i d a d e ~ F e d e r a l ~ d o ~ R i o ~ d e ~ J a n e i r o, ~ R i o ~ d e ~ J a n e i r o, ~ R J, ~ B r a s i l ~}$ \\ ${ }^{\mathrm{b}}$ Universidade Federal do Rio de Janeiro. Rio de Janeiro, RJ, Brasil
}

\section{Histórico do Artigo \\ Recebido em: \\ 03/09/2019 \\ Aceito em: \\ 02/12/2019}

Palavras-chave:

Análise de Redes

Sociais; Referência e

Contra Referência;

Programa Brasil

Sorridente;

governança

\section{RESUMO}

O Programa Brasil Sorridente possui o objetivo de mitigar as desigualdades no acesso à assistência em saúde bucal a todas as faixas etárias nos diversos níveis de atenção, tendo os Centros de Especialidades Odontológicas (CEO) com a função de suprir a demanda por atendimentos especializados. Usuários que chegam às Unidades Básicas de Saúde (UBS) são referenciados aos CEO e posteriormente contra referenciados às UBS de origem, configurando a rede de referência e contra referência (RCR), situadas entre as áreas programáticas do município do Rio de Janeiro. Com o objetivo de demonstrar a aplicabilidade do instrumental teóricometodológico da Análise de Redes Sociais (ARS), avaliou-se a possibilidade de utilização deste instrumental como suporte analítico à governança para futuras investigações da realidade. Com caráter descritivo e analítico, foram pesquisados dados de acesso livre disponíveis no portal do DataSUS. Utilizou-se o software Gephi para a construção dos gráficos da rede formada entre as UBS e CEO. Observaram-se clusters em algumas áreas programáticas, podendo estar trazendo sobrecarga nos atendimentos dos mesmos. Diante da limitação de análise sugere-se que a utilização da ARS, possa ser uma alternativa à governança em prol de programas de saúde.

Center for Dental Specialty and Basic Health Unit: Social Network Analysis as a governance strategy
Keywords:

Social Network

Analysis; Reference and Counter

Reference; Smiling

Brazil Program;

governance

\section{ABSTRACT}

The Smiling Brazil Program aims to alleviate inequalities in access to oral health care for all age groups at the various levels of care, with the Centers for Dental Specialties by the function to meet the demand for specialized care. Patients arriving at the primary heatlh care unity (UBS) are referred to the CEO, and then counter referred to the original UBS, configuring a reference and counter-reference network (RCR). This network is composed by the programmatic areas of the municipality of Rio de Janeiro. The study aimed demonstrating the applicability of the theoretical and methodological tools of the Social Network Analysis (SNA), it was evaluated the possibility of using this instrument as an analytical basis for the governance for future investigations of reality. In this descriptive and analytical research, were researched for free access data available on the DataSUS portal. Gephi software was used to construct the graphs of the network formed between the UBS and CEO. Clusters were observed in some programmatic areas, which may be causing an overload in their services. Even the limitation of analysis, it is suggests that SNA may be an alternative tool to help governance in favor of health programs.

\section{Introdução}

Durante anos a Odontologia esteve à margem das políticas públicas de saúde. O modelo de assistência odontológica estatal vigente era caracterizado por atendimentos a grupos prioritários (crianças em idade escolar) e urgências, gerando um quadro de intensa exclusão do acesso e baixo impacto sobre os índices epidemiológicos de doenças bucais (1).

Visando ampliar a inserção da saúde bucal no Sistema Único de Saúde (SUS), foi lançada, em 2004, a Política Nacional de Saúde Bucal (PNSB), conhecida como Programa Brasil Sorridente (PBS). Esta apresenta diretrizes à ampliação e qualificação

\footnotetext{
* Autor correspondente: fabiorocha2008@gmail.com (de Paula F.R.)
} 
da atenção básica em saúde bucal, através da realização de atividades de promoção, prevenção, assistência e reabilitação, possibilitando o acesso a todas as faixas etárias. Propõe atendimento na atenção secundária, através da implantação dos Centros de Especialidades Odontológicas (CEO), incumbindo-se de assegurar a retaguarda da atenção básica e aumentando seu potencial resolutivo (2,3), tornando o PBS como uma das maiores políticas públicas de saúde bucal do mundo (4).

Sendo a integralidade um dos pressupostos do PBS, deve articular continuamente os serviços e ações preventivos e curativos, individuais e coletivos em todos os níveis de complexidade do sistema (5). O fluxo entre estes níveis de complexidade se realiza por meio do processo da referência e contra referência (RCR). Este fluxo de encaminhamento do paciente adstrito a uma Unidade Básica de Saúde (UBS) é condicionante à sequência prescrita de tratamento deste aos centros de atenção especializada, neste caso os CEO. O fluxo contrário, ou seja, o retorno do paciente à sua UBS de origem, denominado contrarreferência $(6,7)$ compõe tal processo. Certamente, a existência deste procedimento não é garantia de alcance da integralidade, já que não prescindem de outros elementos disponíveis nos serviços da rede de atendimento. Entretanto, é a partir desse processo de RCR que o paciente é inserido nesta rede de atendimento. Advêm desta lógica estruturante, portanto, a premente e regular análise de desempenho do processo de RCR nas redes supra municipais, já que à estas compete oferecer de maneira coordenada, ações que incluam os diversos serviços e garantam a continuidade do cuidado (8). Pois entende-se que para alcançar uma organização articulada e integrada das ações e serviços de saúde, ofertados entre os níveis de atenção do sistema, é mister a estruturação das Redes de Atenção à Saúde (RAS) (9).

As RAS podem ser definidas como organizações poliárquicas, que apresentam pontos vinculados entre si e objetivos comuns, por meio de ações cooperativas e interdependentes, permitem ofertar uma atenção contínua e integral à determinada população, sob coordenação da atenção básica à saúde. Consequentemente o sistema em rede organiza-se sob a horizontalidade de atenção à saúde e tem por centro de comunicação a atenção primária $(10,11)$, tendo a RCR como um dos elementos preponderantes e constitutivos à organização de demanda e constituição das RAS.

Diante disso, a Saúde Bucal deve estar inserida na RAS para a efetiva organização de um modelo de atenção que tenha por objetivo a integralidade do cuidado, estando sincronizada e articulada entre seus níveis (11) e, neste caso representadas aqui entre CEO e UBS. Dado que o procedimento da RCR é obtido por meio da conexão entre serviços, é pertinente analisá-lo através da teoria da Análise de Redes Sociais (ARS). Tal abordagem permite analisar o mecanismo da RCR em si, bem como sua relevância à governança. A ARS é uma teoria, tradicionalmente utilizada com o objetivo de estudar as redes sociais, sejam aquelas ditas "invisíveis" (12), informais, espontâneas e não intencionais oriundas das inter-relações em sociedade, ou aquelas de caráter formal (associações, organizações, clubes, cooperações...), ou seja, permite analisar a estrutura das ligações existentes entre "nós" que compõem as redes sociais $(13,14)$. A ARS em si, pode ser usada como um instrumento indutivo da investigação social, que possuindo o propósito de identificar os tipos de relações existentes entre os atores, mede de forma sistemática os comportamentos relacionais desses atores (15). Medir tais relações, ou a troca de recursos, permite compreender as interdependências e assimetrias existentes em toda ação coletiva (15), sendo, portanto, um possível instrumento de auxílio à governança. A utilização analítica da governança permite que se descreva e explique as interações entre atores, processos e regras, permitindo a compreensão das condutas e tomada de decisões na sociedade (16), mostrando compatibilidade à ARS.

$\mathrm{Na}$ área da Saúde Coletiva brasileira e internacional, o interesse pela ARS vem 
garantindo resultados promissores sejam na avaliação de serviços hospitalares (17), performances de equipes de programas de saúde (18), no campo da gestão e das políticas públicas em saúde (19), na área da saúde mental (20), atenção aos usuários de drogas (21) e até mesmo na relação entre tuberculose e multimorbidade (22). Contudo, ainda que a ARS esteja conquistando credibilidade no campo da Saúde Coletiva brasileira, é oportuno considerar sua importância para a governança, consolidando-a enquanto debuta no contexto acadêmico das práticas avaliativas nos programas de saúde (23).

Governança é um conceito amplo com distintas matizes no campo das políticas públicas (24), caracteriza-se por relações de interdependência, mediada por regras, entre atores diversos em situação de relativa autonomia, sem controle predominante ou subordinação formal, a depender do grau de influência e recursos disponíveis (25). Apesar da convivência com regras formais e marcos legais a governança pode ultrapassar tais regras e expressar a própria natureza dos laços de confiança e das estratégias de coordenação e negociação de interesses em cada contexto (26).

A governança depende, portanto, do desenho institucional e da interlocução com os grupos organizados da sociedade para definição, acompanhamento e implementação de políticas públicas (27). Neste aspecto nos aproxima dos processos e governança em saúde, uma vez que, pelo pacto federativo, a implementação do SUS em nível local depende do estabelecimento dessas relações (24). Pode, como a ARS, ser usada como ferramenta analítica para investigação e transformação dos processos de formulação de políticas e gestão de sistemas de saúde $(28,29)$.

O presente artigo pretende demonstrar a aplicação do instrumental teóricometodológico pertinente a ARS como suporte à governança, a partir do Programa Brasil Sorridente do município do Rio de Janeiro, conforme o preconizado para o processo de RCR entre UBS e CEO em suas respectivas Áreas Programáticas (AP). Desta forma, os dados aqui utilizados resultam na rede de RCR teórica preconizada, ou seja, a ideal, que, servindo de base analítica, dará suporte a futuras investigações da realidade.

\section{Materiais e métodos}

Este artigo propõe a aplicação da teoria da ARS, com a finalidade de demonstrar que o instrumental teórico-metodológico pertinente a essa temática pode ser utilizado como suporte à governança. Utilizou-se de dados de acesso livre, encontrados no site oficial do Portal da Saúde do Sistema Único de Saúde (SUS) (www.portalsaude.saude.gov.br), através do item serviços, onde é acessado o Programa Brasil Sorridente. No site do programa buscou-se em: Atenção especializada; Centro de Especialidades Odontológicas; Cidades Atendidas e por fim através do mapa do Rio de Janeiro. Mediante o qual foi realizado o acesso às informações relativas ao município do Rio de Janeiro de cujas áreas programáticas possuem CEO, com seus respectivos códigos e números de Cadastro Nacional de Estabelecimentos de Saúde (CNES). O período da pesquisa ocorreu durante todo o mês de setembro de 2018. Utilizou-se como referencial os dados da população do município do Rio de Janeiro, do Censo de 2010 do Instituto Brasileiro de Geografia e Estatística (IBGE), sendo este o mais recente até o momento, pois o mesmo ocorre a cada 10 anos.

Foram encontrados 18 CEO situados no município do Rio de Janeiro. Conforme o seu número do CNES identificou-se a localização dos bairros onde eles atuam, por Área Programática (AP). O município do Rio de Janeiro possui 160 bairros, agrupados entre as 10 áreas programáticas e, estas foram divididas desde o ano de 1993 pela Secretaria Municipal de Saúde (SMS), no intuito de melhorar o gerenciamento dos serviços de saúde. As áreas programáticas possuem suas particularidades em função da história e evolução da ocupação da cidade, determinando características regionalizadas com 
relação à atividade econômica, mobilidade, serviços de saúde, educação, as formas de adoecimento e mortalidade (30).

As informações dos endereços dos CEO foram obtidas através do site: cnes.datasus.gov.br. E os endereços das UBS foram adquiridos no site da Prefeitura do Rio de Janeiro em: www.rio.gov.rj, seguindo os links disponíveis na página: $A$ prefeitura; órgãos municipais; secretarias; SMS; unidades de saúde e, por fim onde ser atendido. Neste é disponibilizado o mapa com a visualização das 223 UBS disponíveis, por suas respectivas áreas programáticas.

O atendimento inicial em saúde bucal aos usuários entre as 223 UBS é ofertado em seu território de referência, onde os mesmos acessam o link "onde ser atendido", através do endereço eletrônico: https://smsrio.org/subpav/ondeseratendido/. O atendimento especializado é realizado, após avaliação inicial do paciente pelo cirurgião-dentista da UBS (Atenção Primária), através de encaminhamento via Sistema de Regulação de vagas (SISREG) para um dos Centros de Especialidades Odontológicas (CEO) ou hospital especializado, preferencialmente próximo ao território de domicílio do paciente (territorialização), de acordo com as informações disponíveis no site da Prefeitura do Rio de Janeiro, em: http://www.rio.rj.gov.br/web/sms/saude-bucal.

Assim, a partir dos dados obtidos nos referidos endereços eletrônicos e base de dados, foram elaborados grafos para a visualização do processo de RCR utilizando o software Gephi, de acesso livre, em sua versão 0.9.1. Os dados foram organizados em planilha Excel/Windows, como lista de adjacências (constituída por uma planilha Excel onde as relações a partir de cada nó são colocadas em linhas separadas por vírgulas, permitindo a identificação dos mesmos com os demais adjacentes). Denomina-se nó aos atores que compõem a rede. Podem representar pessoas, instituições, serviços, etc. (21). Nessa pesquisa, os CEO foram identificados como os nós de CEO1 até CEO18. As relações dos nós seguem em cada linha, com as UBS de acordo com a área programática pertencente. As UBS estão identificadas como n1 até n223. O indicador de ocorrência de relação entre uma UBS e um CEO, e vice-versa, foi a distribuição apresentada por cada uma das AP, conforme preconizado pelo SUS, sendo a Atenção Básica a porta de entrada responsável pelo atendimento da população adstrita (31). A lista resultante foi salva em Excel no formato $C S V$ e posteriormente aberta no software Gephi sob o algoritmo Fruchterman Reingold, produzindo os sociogramas. Estes apresentam a visualização das relações entre os nós (CEO e USB), representando a rede de RCR conforme as especificações idealizadas pela lógica da descentralização e atendimento em rede do SUS. Portanto, trata-se da visualização da rede como deveria ser, e não a real.

\section{Resultados}

O quadro 1 apresenta os Centros de Especialidades Odontológicas em suas respectivas áreas programáticas, relacionadas com as UBS representadas pelos códigos (n1 ao n 223). 
Quadro 1 - Relação dos Centros de Especialidades Odontológicas e Unidades Básicas de Saúde pelas Áreas programáticas no município do Rio de Janeiro

\begin{tabular}{|c|c|c|}
\hline $\begin{array}{c}\text { ÁREAS } \\
\text { PROGRAMÁTICAS }\end{array}$ & $\begin{array}{c}\text { CENTRO DE } \\
\text { ESPECIALIDADES } \\
\text { ODONTOLÓGICAS }\end{array}$ & $\begin{array}{c}\text { UNIDADES BÁSICAS DE } \\
\text { SAÚDE }\end{array}$ \\
\hline AP 1.0 & CEO 1 e CEO 2 & n1 a n18 (18 UBS) \\
\hline AP 2.1 & $\mathrm{CEO} 3$ e CEO 4 & n19 a n31 (13 UBS) \\
\hline AP 2.2 & CEO 5 & n32 a n40 (9 UBS) \\
\hline AP 3.1 & CEO 6, CEO 7 e CEO 8 & n41 a n74 (34 UBS) \\
\hline AP 3.2 & CEO 9 e CEO 10 & n75 a n97 (23 UBS) \\
\hline AP 3.3 & CEO 11 e CEO 12 & n98 a n126 (29 UBS) \\
\hline AP 4.0 & CEO 13 e CEO 14 & n127 a n144 (18 UBS) \\
\hline AP 5.1 & CEO 15 & n145 a n167 (23 UBS) \\
\hline AP 5.2 & CEO 16 e CEO 17 & n168 a n198 (31 UBS) \\
\hline AP 5.3 & CEO 18 & n199 a n223 (25 UBS) \\
\hline
\end{tabular}

Fonte: própria

De acordo com o quadro 1, confirma-se que o procedimento de RCR é proposto apenas para as áreas programáticas onde existem CEO (dezoito, no total) e UBS (223, no total). Porém 10 CEO também atuam como UBS, realizando duplo papel, de referência e contra referência de si próprio.

A figura 1 representa o sociograma do modelo teórico conforme a sistematização dos dados aqui realizada. Observam-se as relações da RCR entre os CEO e as UBS. As UBS encaminham os usuários para os CEO dentro de suas áreas programáticas e estes contra referenciam os usuários para as UBS de origem, dando continuidade aos tratamentos. Percebe-se que nas áreas programáticas onde os CEO também atuam como UBS há maior número ligações.

As áreas programáticas AP 3.1, AP 3.3 e AP 5.2, apresentam maior número de UBS e CEO e, por conseguinte possuem maior quantidade de relações (102, 58 e 62, respectivamente). As distribuições, entre os UBS e CEO, pelo território não são homogêneas. Portanto, em outras áreas programáticas, as relações se dispõem com menor número de arestas (ligações entre os nós). Tal fato ocorre devido a disponibilidade de nós por áreas, caracterizando-se por regiões com alta densidade (quanto maior o número de ligações entre os nós, mais densa será a rede) e centralidade de grau (relacionado ao nó que está diretamente ligado a muitos outros, próximo a 100\%). Já nas áreas programáticas AP 2.1 e AP 2.2, onde ocorrem 26 e 9 relações respectivamente, foram observadas redes com coesão (referente às características de estrutura e organização da rede) de densidade e centralidade de grau de CEO inferiores, quando comparados aos CEO das áreas programáticas AP 3.1, 3.3 e 5.2. Verifica-se ainda na figura 1 uma característica marcante, os clusters ou cliques (conjunto de 3 ou mais nós interligados entre si, formando subgrupos dentro da rede) constituídos pelas UBS nas proximidades dos CEO. 
Figura 1 - Rede de relações entre os CEO e UBS do município do Rio de Janeiro

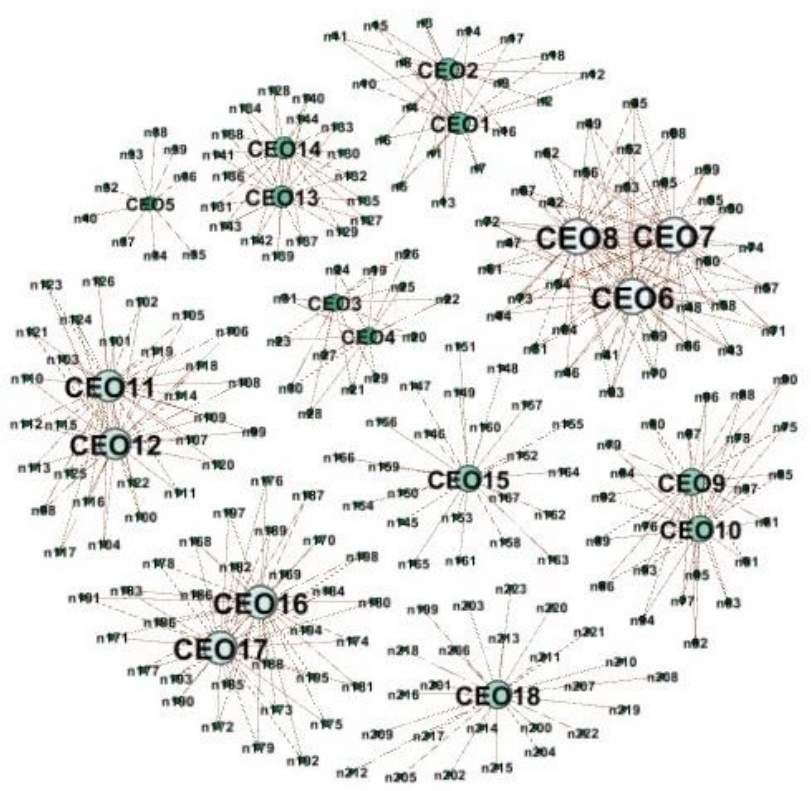

A seguir, a figura 2 apresenta os mesmos dados, dispostos em sociograma sobreposto ao mapa do município do Rio de Janeiro, facilitando a visualização das relações preconizadas entre as UBS e CEO, nas áreas programáticas. As ligações, ou arestas, representadas pelas linhas, indicam os percursos propostos para RCR das UBS para os CEO e, vice-versa, por regiões adstritas. As arestas estão restritas às suas áreas programáticas, ou seja, cada UBS se dirigindo ao CEO pertencente a sua área programática. Também, no mapa da Figura 2, é possível visualizar a localização dos clusters das áreas programáticas (AP 1.0; AP 2.1; AP 3.1; AP 3.2; AP 3.3; AP 4.0 e AP 5.2) evidenciados na figura 1, em relação ao espaço geográfico relativo à população adstrita.

Figura 2 - Mapa do município do Rio de Janeiro, suas áreas programáticas e relações entre os CEO e UBS.

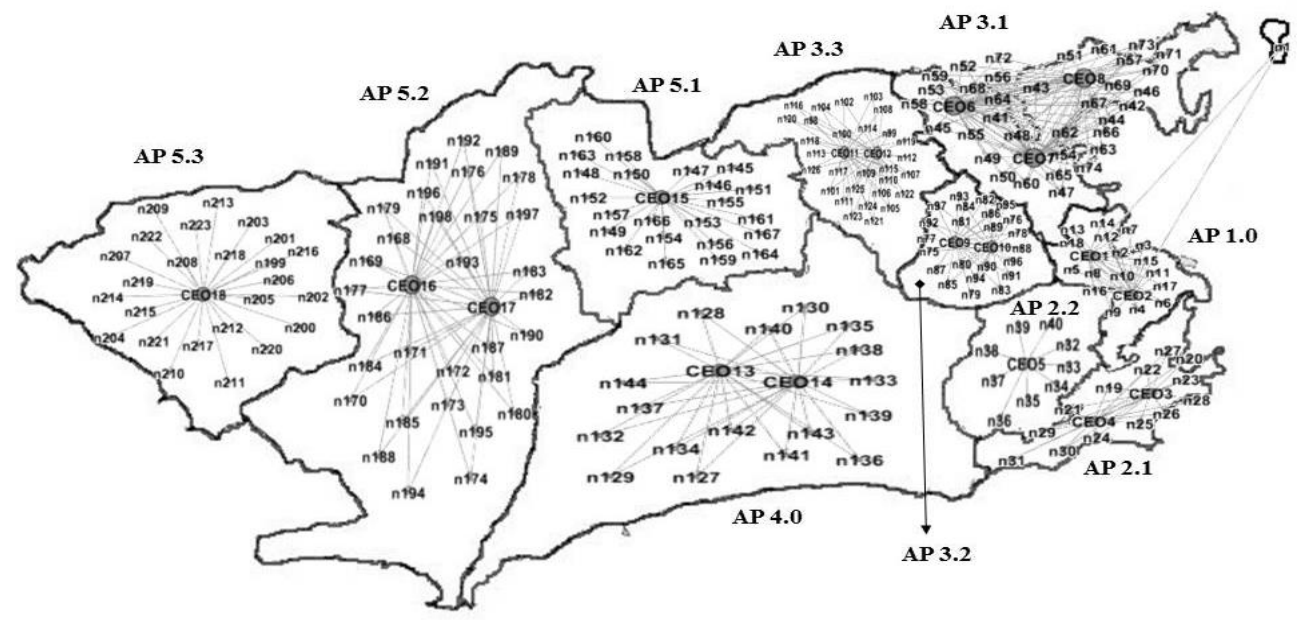

\section{Discussão}

A partir da inserção dos dados, no software Gephi para elaboração de redes, foi possível visualizá-los graficamente, correspondendo tais imagens à dinâmica preconizada pela PNSB. Ou seja, representam a materialização visual da rede pretendida, ensejando prospecções futuras e facultando a aplicação da ARS não só aplicada a processos avaliativos, mas também ao planejamento e decisão. 
Considerando que a proposta dos procedimentos de RCR apresenta-se, de fato, como uma rede, sendo seus nós os CEO e as UBS e suas relações, os encaminhamentos preconizados entre eles, a análise visual deste sistema permite avaliar possibilidades e desafios à PNSB.

O município do Rio de Janeiro, com suas UBS e CEO distribuídos entre as 10 áreas programáticas, conforme a figura 2 apresenta três AP (AP 2.2, AP 5.1 e AP 5.3), as quais possuem apenas um CEO cada uma delas (CEO 5, CEO 15 e CEO 18, respectivamente). Esses CEO possuem alto grau de centralidade em relação aos seus nós (UBS). Como a centralidade representa o número de ligações relacionadas a determinado nó, quanto maior a quantidade de ligações, maior a centralidade (32). Sendo a centralidade a medida das ligações de atividade de um nó, ou ator na rede $(33,32)$, esta facilita a descrição de eventos e organização dos elementos (34).

Neste caso, importa verificar se a relação da demanda e capacidade de suporte destes três CEO está compatível com a população local onde os mesmos se encontram. Pois o município do Rio de Janeiro, apresenta uma extensão territorial de $1.200 .179 \mathrm{Km}^{2}$ e uma população de 6.320.446 habitantes, de acordo com o Censo de 2010 do IBGE. Partindo desse pressuposto verificam-se por exemplo que as AP 3.3 e AP 4.0 possuem dois CEO cada uma e, são as áreas com as maiores distribuições populacionais do município do Rio de Janeiro, com 959.404 e 919.420 habitantes respectivamente (30), tal fato evidentemente não fará jus à população usuária destas regiões situadas nessas áreas citadas.

$\mathrm{Na}$ figura 1, são observados clusters de $\mathrm{CEO}$, visualizados entre as áreas programáticas $2.1 ; 3.1 ; 3.2 ; 3.3 ; 4.0$ e 5.2. Estes CEO (CEO 3, 4, 7, 8, 9, 11, 13, 14, 16 e 17) são aqueles que cumprem também o papel duplo e, por este aspecto entende-se que podem estar funcionando como porta de entrada no sistema (35-37). Como Clusters, remetem a quaisquer tipos de concentrações em uma rede (38), sugerem maior possibilidade de movimentação, que influenciam sua dinâmica (39-41). Entretanto, sendo os CEO, serviços de referência, têm obrigatoriamente papel de convergência, mesmo que temporária. Esta condição propicia situações de demandas pouco controláveis. Por exemplo, de acordo com Censo demográfico de 2010 do IBGE, as populações residentes nessas áreas com apenas um CEO que são a AP 2.2 (CEO 5 constituída por 7 bairros num total de 375.221 habitantes); a AP 5.1 (CEO 15 constituída por 9 bairros num total de 678.457 habitantes) e a AP 5.3 (CEO 18 constituída por 3 bairros num total de 372.607 habitantes), provavelmente podem estar sujeitas a um longo tempo de espera entre agendamentos, se comparadas aos locais com clusters de CEO.

Em outras palavras, mesmo que apenas uma pequena parcela das pessoas - $14,2 \%$ de cobertura populacional potencial - por equipe de saúde bucal (eSB) tenha necessidade destes serviços especializados, e estimando-se que a eSB responsabilize-se por 3.000 habitantes (42), a oferta e a localização dos CEO remetem às dificuldades ao usuário. Mesmo que para a equipe de Saúde da Família, o preconizado seja o atendimento de, no máximo, 4.000 pessoas (43), esta tem atuações e números de equipes diversas da eSB.

Ainda considerando que entre 2002 e 2015 o número de equipes de saúde bucal foi ampliado a mais de $467 \%$ em todo o Brasil (44). Tal aumento foi desigual entre as regiões do país. No caso do município do Rio de Janeiro, mesmo com a implantação e o crescimento do número de equipes de saúde bucal ocorridas de forma expressiva, após o ano de 2009 (45), a análise das figuras 1 e 2, descreve uma distribuição e alocação de equipes heterogêneas, nesse momento.

Pelo mapa é fácil verificar que a estratégia RCR exige como requisito mister, o estabelecimento da rede entre UBS e CEO, conforme o preconizado, com centralidade 
dos CEO, porém evitando-se os clusters. Nisto, o planejamento minucioso da rede de RCR, deve ter papel preponderante. Com a utilização da ARS, as dificuldades e necessidades podem ser previstas e evitadas, ou identificadas e solucionadas. Prevê-las, superá-las e/ou amenizá-las, pode encurtar o caminho para a resolução das necessidades em saúde bucal (46). Requerem políticas de integração de ações preventivas, curativas e de reabilitação, responsabilidade de todos os segmentos sociais e compromisso do Estado (47). Ou seja, está intrinsecamente ligada à governança, de modo a preestabelecer padrões de articulação e cooperação entre atores sociais e políticos, prever arranjos institucionais que coordenem e regulem transações dentro e através das fronteiras do sistema, assim como fomentar a articulação de interesses mútuos, como também estimular redes sociais informais e associações de diversos tipos (48). Como a governança envolve modos de exercício de autoridade e processos de inserção dos interesses sociais na agenda do governo, é ferramenta analítica inolvidável na investigação e transformação dos processos de formulação de políticas e gestão de sistemas de saúde $(28,29)$. De fato, pois a governança implica na definição de princípios, normas e balizas para o processo decisório de maneira dinâmica e plural em diferentes campos da política pública. Articulando-se com o debate sobre a natureza e a efetividade das iniciativas de promoção de saúde (26).

Como ARS explora a dimensão de variáveis além das tradicionais, baseando-se nas diferenças entre pessoas, momentos e lugares (22), ao servir de apoio à prática da governança, concorrerá à marcantes resultados em todos os setores da saúde. Assim, a visualização das figuras 1 e 2, evidenciando o quão importante é a centralidade na lógica da RCR, faz refletir acerca dos desafios invisíveis, por inexistência de instrumento capaz de lhes tornar perceptíveis.

Os serviços têm dificuldades em se comunicar, desenvolvendo lógicas próprias e distintas (49). Nesta perspectiva, a integração da atenção primária com a especializada, apesar de fundamental, é agravante para a RCR, a qual aumenta a demanda por tratamento especializado (50). Para evitar este tipo de problema é imprescindível analisar sistematicamente os modelos de gestão propostos, incluindo a ampliação da capacidade dos governos na implementação das políticas públicas como estratégia de promoção da justiça social (51).

Destarte, a possibilidade de visualização da organicidade da RCR vem a se constituir importante instrumento de governança. E, mesmo considerando as limitações desta análise, é possível ponderar que os CEO em desempenho de dupla função, possam estar com deficiências de equipes, dada a demanda do próprio público de usuários como a de usuários na área adstrita, aí acumulada. Essa capacidade de previsão conseguida por meio da simples visualização geral do cenário, viabilizada pela utilização da abordagem da ARS, mostra-se elemento facilitador para a governança.

\section{Conclusões}

Diante do exposto, verifica-se que a utilização do instrumental da ARS como estratégia para planejar cenários, avaliar possibilidades e analisar situações consolidadas é pertinente e inovadora. Apesar das limitações relativas a natureza dos dados aqui empregados, foi possível, mesmo assim, constatar o grande potencial de utilização da ARS como auxilio a governança, uma vez que gráficos têm a capacidade de informar visualmente um contexto em seu conjunto, quesito fundamental à governança. $\mathrm{O}$ emprego da RCR como parâmetro, baseado em uma única métrica, a da centralidade, dentre as possíveis em ARS, viabilizou verificar-se a distribuição e conexões das UBS com a atenção especializada (CEO), demonstrando-se a potência da ARS para a 
governança. Nesta perspectiva a utilização da ARS pode ser válida, como ferramenta analítica de suporte aos processos de formulação de políticas públicas e na gestão de uma rede regionalizada e integrada como o Programa Brasil Sorridente.

\section{Referências}

1. Baldani MH. A experiência em cidades de médio e pequeno porte do Paraná. In: Moysés ST, Kriger L, Moysés SJ, (Org). Saúde bucal das famílias: trabalhando com evidências. São Paulo: Artes Médicas; 2008.

2. Silva KL, Sena RR. Integralidade do cuidado na saúde: indicações a partir da formação do enfermeiro. Rev Esc Enferm USP 2008; 42(1): 48-56.

3. Bulgareli JV, Faria ET, Ambrosano GMB, Vazquez FL. Informações da Atenção Secundária em Odontologia para avaliação dos modelos de atenção à saúde. Rev de Odonto da UNESP 2013; 42(4): 229-36.

4. Pucca GA Jr, Gabriel M, de Araujo ME, de Almeida FC. Ten Years of a National Oral Health Policy in Brazil: Innovation, Boldness, and Numerous Challenges. J Dent Res 2015; 94(10): 1333-7.

5. Fratini JRG, Saupe R, Massaroli A. Referência e Contra Referência: Contribuição para a Integralidade em Saúde. Ciênc Cuid Saúde 2008; 7(1): 65-72.

6. Acurcio FA, Cherchiglia ML, Santos MA. Avaliação de qualidade de serviços de saúde. Saúde Debate 1991; 33:50-3.

7. Rodrigues LA, Vieira JDM, Leite ICG. Avaliação do fluxo de referência para um centrode especialidades odontológicas implantadoem cidade de médio porte na região Sudeste. Cad Saúde Colet 2013; 21(1):40-5.

8. Lima JC. Território situado, redes de conversação e coordenação: novas perspectivas para a integralidade da atenção à saúde. In: Ferreira SCC, Monken M, Organizadores. Gestão em saúde: contribuições para a análise da integralidade. Rio de Janeiro: EP-SJV; 2009. p. 103-27.

9. Lavras C. Descentralização, regionalização e estruturação de redes regionais de atenção à saúde no SUS. In: Ibanez N, Elias PEM, Seixas PHD, organizadores. Política e Gestão Pública em Saúde. São Paulo: Hucitec Editora: Cealag, 2011.

10. Mendes, EV. As redes de atenção à saúde. Brasília: Organização Pan-Americana da Saúde; 2011.

11. Gondinho BVC, Guerra LM, Bulgareli JV, Probst LF, Cortellazzi KL, Possobon RF et al. Percepção de coordenadores de saúde bucal sobre a rede de atenção à saúde bucal. Rev Bras Promoç Saúde 2018; 31(Supl): S1-8.

12. Grossetti M. Sociologie de l'imprevisible. Dynamiques de l'activitéetdes formes sociales. Paris: PUF Collection, 2004. 225p.

13. Grossetti M. Dynamiques des réseaux et des cercles. Encastrementset découplages. Revue d'économie industrielle 2003; 2: 327-55.

14. Tomael MI, Marteleto RM. Redes sociais de dois modos: aspectos conceituais. Transinformação 2013; 25(3): 245-53.

15. Varanda M. Acção colectiva entre pequenos empresários: uma análise de redes sociais. Análise Social 2007; 42(182): 207-30.

16. Hufty M, Báscolo E, Bazzani R. Gobernanza en salud: un aporte conceptual y analítico para la investigación. Cad Saúde Publica 2006; 22(Supl): S35-45.

17. Bittencourt NOS, Neto FJK. Rede social no sistema de saúde: um estudo das relações interorganizacionais em unidades de serviços de HIV/AIDS. Revista de Administração Contemporânea 2009; 13:87-104.

18. Gomide M, Grossetti M. Rede social e desempenho de programas de saúde: uma proposta investigativa. Physis Rev Saúde Colet 2010; 2 (3): 873-93.

19. Lino C, Gomide M. Análise de redes sociais na avaliação do programa de controle de hanseníase em um município do interior do Brasil. Cad Saúde Colet 2012; 20(1): 32-40.

20. Salles MM, Barros S. Inclusão social de pessoas com transtornos mentais: a construção de redes sociais na vida cotidiana. Ciên Saúde Colet. 2013; 8(7): 2129-38.

21. Costa PHA, Ronzani TM, Colugnati FAB. Análise de Redes Sociais: Uma Estratégia Avaliativa para 
a Rede de Atenção aos Usuários de Álcool e outras Drogas. Psicologia e Saúde Pública: Contribuições e Desafios. 2015:227-246 Editora UFJF, Juiz de Fora.

22. Valenzuela-Jiménez H, Manrique-Hernández EF, Idrovo AJ. Relação da tuberculose com multimorbidade e redes sociais. J Bras Pneumol. 2017; 43 (1): 51-53.

23. Gomide M, Schütz GE. Análise de Redes Sociais e práticas avaliativas: desafios à vista. Physis Rev Saúde Colet 2015; 25(3): 819-42.

24. Casanova AO, Giovanella L, Cruz MM, Ferreira MFDC. Atores, espaços e rede de políticas na governança em saúde em duas regiões de saúde da Amazônia Legal. Ciên Saúde Colet 2018; 23(10): 3163-3177.

25. Fleury S, Ouverney ALM. Gestão De Redes: A Estratégia De Regionalização da Política de Saúde. Rio de Janeiro: FGV; 2007.

26. Magalhães R. Governança, redes sociais e promoção da saúde: reconfigurando práticas e institucionalidades. Ciên Saúde Colet 2018; 23(10): 3143-3150.

27. Azevedo S, Anastácia F. Governança, “accountability” e responsividade. Rev Econ Polit 2002; 22(1): 79-97.

28. Fleury S, Ouverney ALM, Kronemberger TS, Zani FB. Governança local no sistema descentralizado de saúde no Brasil. Rev Panam Salud Publica 2010; 28(6): 446-55.

29. Santos AM, Giovanella L. Governança regional: estratégias e disputas para gestão em saúde. Rev Saúde Pública 2014; 48(4): 622-31.

30. SMS. Secretaria Municipal de Saúde. Plano Municipal de Saúde do Rio de Janeiro - PMS (20142017). 2013. Rio de Janeiro: Secretaria Municipal de Saúde. Disponível em: <www.rio.rj.gov.br/web/SMS>. Acesso em: $01 \mathrm{dez} .2017$.

31. Brasil. Portaria n 2.488, de 21 de outubro de 2011. Aprova a Política Nacional de Atenção Básica, estabelecendo a revisão de diretrizes e normas para a organização da Atenção Básica, para a Estratégia Saúde da Família (ESF) e o Programa de Agentes Comunitários de Saúde (PACS). Diário Oficial [da República Federativa do Brasil], Brasília, n. 204, p. 55, 24 out. 2011. Seção 1, pt1.

32. McCarty C. La estructura en las redes personales. Redes - Rev Hispana Para el Anal de Redes Sociales 2010; 19(11): 242-71.

33. Degenne A, Forse M. Les Réseaux Sociaux. Paris: Armand Colin, 2004. 295p.

34. Borgatti SP, Everett MG. Graph-theoric perspective on centrality. Soc Networks 2006; 28: 466-84.

35. Kimatura ES, Bastos RR, Palma PV, et al. Avaliação da satisfação dos usuários dos Centros de Especialidades Odontológicas da macrorregião Sudeste de Minas Gerais, 2013. Epidemiol Serv Saúde 2016; 25(1): 137-148.

36. Thomaz EBAF, Sousa GMC, Queiroz RCS, et al. Avaliação do cumprimento das metas de produtividade em Centros de Especialidades Odontológicas no Maranhão, 2011. Epidemiol Serv Saúde 2016; 25(4): 807-818.

37. Rios LRF, Colussi CF. Avaliação normativa dos Centros de Especialidades Odontológicas, Brasil, 2014. Saúde Debate 2019; 43(120): 122-136.

38. Régis HP, Bastos AVB, Dias SMRC. Redes sociais informais: análise das redes de amizade, de informação e de confiança em incubadoras de base tecnológica no Recife. Rev Psic Org e Trabalho 2007; 7(1): 31-56.

39. Hannemann RA. Introducción a los métodos del análisis de redes sociales. Departamentode Sociología de la Universidad de California Riverside. Redes. 2000; p. 1-9 Disponível em: <revistaredes.rediris.es/webredes>. Acesso em: 10 dez. 2012.

40. Marteleto R. Análise de Redes Sociais: aplicação nos estudos de transferência da informação. Cien. Inf 2001; 30(1): 71-81.

41. Portugal S. Quanto vale o capital social? O papel das redes informais na provisão de recursos. In: Fontes B, Martins PH. (Org.). Redes, práticas associativas e gestão pública. Recife: Ed Universitária UFPE, 2006. p. 51-74.

42. Lima Júnior, IA. Estudo sobre a Coordenação Assistencial entre os Serviços de Atenção Primária e Especializados em Saúde Bucal da Área de Planejamento 3.1 da Cidade do Rio de Janeiro. [Dissertação]. Rio de Janeiro: Escola Nacional de Saúde Pública Sérgio Arouca. Fundação Oswaldo Cruz. Fiocruz; 2016. 
43. Brasil. Ministério da Saúde. Estratégia Saúde da Família (ESF). s/d. Disponível em: <http://portalms.saude.gov.br/acoes-e-programas/saude-da-familia/sobre-o-programa>. Acesso em: 23 nov. 2017.

44. Brasil. Ministério da Saúde. Secretaria de Atenção à Saúde. Departamento de Atenção Básica. Coordenação Nacional de Saúde Bucal. Conheça a Política que faz muitos brasileiros voltarem a sorrir. Brasília/DF, 2015.

45. Casotti E, Gabriel JO. Organização da Atenção em Saúde Bucal na Cidade do Rio de Janeiro: Resultados do Programa de Melhoria do Acesso e Qualidade da Atenção Básica. Rev APS 2016; 19(2): 245-60.

46. Vazquez FL, Guerra LM, Vítor ES, Ambrosano GMB et al. Referência e Contrarreferência na Atenção Secundária em Odontologia em Campinas, SP, Brasil. Ciên Saúde Colet 2014; 19(1): 245-55.

47. Parro YM, Guerrero AVP O Direito humano ao sorriso: uma análise do arcabouço legislativo sobre a saúde bucal. Revista Cadernos Ibero-Americanos de Direito Sanitário 2016; 5(2): 109-30.

48. Santos MHC. Governabilidade, Governança e Democracia: Criação da Capacidade Governativa e Relações Executivo-Legislativo no Brasil Pós-Constituinte. DADOS - Revista de Ciências Sociais 1997; 40 (3) 335-76.

49. Kantorski LP, Jardim VMR, Coimbra VCC, Oliveira MM, Heck RM. A integralidade da atenção à saúde na perspectiva da gestão do município. Texto contexto - enferm 2006; 15(3): 434-441.

50. Aquilante AG, Aciole GG. O cuidado em saúde bucal após a Política Nacional de Saúde Bucal "Brasil Sorridente": um estudo de caso. Ciên Saúde Colet 2015; 20(1): 239-48.

51. Costa e Silva V, Barbosa PR, Hortale VA. Parcerias na saúde: as organizações Sociais como limites e possibilidades na gerência da estratégia Saúde da Família. Ciên Saúde Colet 2016; 21 (5): 1365-76. 